Ludovico Longhi ${ }^{1}$

Universitat Autònoma de Barcelona

Dep. Comunicación Audiovisual
UDK: 316.72"1980"(450)

https://doi.org/10.18485/imp.2017.ch.23

\title{
ALBERTO SORDI, RAI E IDENTIDAD NACIONAL EN LOS OCHENTA
}

Al final de los setenta (los años de plomo, llenos de esperanzas frustradas y de malogrados cambios sociales) la RAI perfecciona un plano estratégico para salvaguardar la identidad nacional. Dicho proyecto, no solo respondía la necesidad de reafirmar un sello propio transalpino en la programación televisiva autóctona, sino también a la introducción de una imagen corporativa dentro del nuevo contexto de liberalización del mercado del éter, donde se multiplicaba la competencia de cadenas privadas y la transmisión de programas extranjeros. Con este propósito la cadena nacional italiana se pone al frente de un proceso de creación para compartir un imaginario colectivo del espíritu nacional, retransmitiendo con gran énfasis toda una serie de eventos que proponían el Made in Italy como garantía de calidad y éxito gracias a los triunfos de los célebres estilistas transalpinos, la positiva conclusión de la misión militar de paz en Líbano y la victoria de la selección nacional en el campeonato mundial de fútbol de 1982 en España. Estas circunstancias positivas contribuyeron a despertar el interés por la definición del concepto de italianidad, entendido como una categoría socio-política, geo-antropológica y meta-histórica. El proyecto, sin embargo, desarrolló un nuevo sentimiento de orgullo nacional que reciclaba en positivo un cúmulo de obsoletos estereotipos del peor nacionalismo. Consecuentemente, puesto que solo una minoría de las características de la italianidad podían convertirse en modelos ejemplares, fue necesario proceder a la revisión de la célebre comedia cinematográfica a la italiana, censurando su alto poder crítico y satírico de las malas y difusas costumbres nacionales. En este artículo es mi intención presentar el caso emblemático de la realización de Storia di un italiano un programa donde Alberto Sordi, a cambio de ser nombrado icono príncipe de la italianidad, intenta reconsiderar la historia italiana contemporánea combinando piezas de su impresionante filmografía con fragmentos de los cine diarios del Istituto Luce. Desafortunadamente dicho collage, realizado en pleno acuerdo con la nueva estrategia de la RAl, acabó desnaturalizando el carácter sarcástico de las creaciones radiofónicas y cinematográficas sordianas.

Palabras clave: Alberto Sordi, RAl, comedia, cine italiano, años ochenta

1 Ludovico.Longhi@uab.cat 


\section{Historia de un italiano atípico}

Justo a finales de la década de los setenta el entusiasta e hiperactivo funcionario de la RAI, Giancarlo Governi (periodista, escritor, autor de programas biográficos de gran éxito ${ }^{2}$ ) convence a Alberto Sordi para que realice Storia di un Italiano: un programa de actualidad (así es definido en la programación) donde el actor romano pretende contar la historia de Italia y los italianos mezclando secuencias de sus películas con fragmentos de los documentales del Instituto Luce. El amor incondicionado de Governi por los años dorados de la cinematografía transalpina y una precisa estrategia de los equipos directivos RAI para hacer frente a la nueva situación de mercado del éter, insisten hasta el convencimiento de que el cómico trastiberino es un prototipo de los italianos (¿y las italianas?), tan verídico, que es posible reinscribir una crónica fiel de los acontecimientos itálicos contemporáneos. Sin embargo no sólo el collage de fragmentos acaba sofocando irremediablemente la gracia y la eficacia de las interpretaciones sordianas, sino que la entera operación llega a un grado de manipulación tan alto que roza la falsedad.

Más que el italiano medio con sus defectos y virtudes, desde sus inicios y a lo largo de los años sesenta Alberto Sordi interpreta en sus películas toda una galería de monstruos en libertad, de personajes repugnantes y siniestros que no buscan ni el consentimiento ni la simpatía del público. Paralelamente en las recensiones cinematográficas relativas a sus personajes, hay un adjetivo recurrente: sórdido, o sea un agudo juego de palabras construido alrededor de su apellido. Pero también asqueroso, repelente, sucio, maligno y una considerable cantidad de símiles epítetos que difícilmente se podrían aplicar a un actor que quiere ser el tipo modélico de sus compatriotas.

En efecto Alberto Sordi desde sus primeras intervenciones ya en los años treinta apostaba por un mecanismo cómico de contra-tenden-

2 Il pianeta Totò, Totò: un altro pianeta, Laurel \& Hardy: Due teste senza cervello, Parlami d'amore Mariù, Mille bolle blu, Italiaride, La leggenda di Mister Volare, la serie Ritratti: sobre las carreras de Albero Sordi, Marcello Mastoianni, Ugo Tognazzi, Renato Rascel, Massimo Troisi, Anna Magnani, Aldo Fabrizi, Domenico Modugno, Maria Callas, Vittorio De Sica, Billi e Riva.... 
cia, totalmente original y arriesgado, que no denunciaba los defectos nacionales (práctica que se convierte en fórmula en la comedia de los sesenta), sino que los encarnaba exasperándolos, que insistía en la diversidad excéntrica, asocial y casi patológica con una ferocidad sorprendente.

Sordi, caso muy complejo e insólito entre los grandes actores cinematográficos, ha sido el único que ha rechazado la idea de que el público se identificase con la máscara que él había creado y esto significó renunciar al mecanismo básico del éxito popular. Sin embargo, a mitad de los cincuenta alcanza la máxima popularidad. Consigue ser el número uno y se mantiene en la cumbre a lo largo de veinte años, sin ninguna condescendencia ni indulgencia, todo lo contrario de sus colegas cómicos. Éstos se las ingenian, se concentran para transformar en simpáticos y atractivos a sus bribones y granujas. Sordi no: él interpreta unos sinvergüenzas sin complacencia; les da vida sin justificarlos. Es a su manera, sincero y despiadado en representar el abismo moral de sus canallas.

Después de la gran visibilidad obtenida gracias a las dos obras maestras de Federico Fellini [sobretodo de I vitelloni (Los inútiles, 1953), más que de Lo sceicco bianco (El jeque blanco, 1952)] Sordi construye y perfecciona su máscara repelente en una serie de películas menores (es decir, películas de episodios o de montaje de distintos números cómicos) donde tiene más libertad para arriesgarse, para experimentar. Trabaja sin parar en un filme detrás de otro (12 entre 1955 y 1956) aumentando cada vez más la dosis de provocación. El examinador meticuloso y despiadado en Totò e i re di Roma (Steno e Mario Monicelli, 1952) es solo la prueba general de futuras impiedades, en apariencia más sainetescas y meno contundentes, pero en el fondo muy insolentes e impertinentes.

La máscara de Albertone se configura como un joven malcriado, consentido y mimado por abuelas, madres y tías excesivamente protectoras en películas como Accadde al penitenziario [La cárcel de los líos (Giorgio Bianchi, 1955)], donde sin motivo molesta a unos noctámbulos, Piccola posta (Steno, 1955) donde maltrata a un grupo de pobres abuelitas, Bravissimo (Luigi Filippo D'amico, 1955) donde se aprovecha de las precoces habilidades canoras de un niño-barítono o Arrivano i dollari! (Mario Costa, 1957) donde interpreta a un impunito uxoricida que trata a su mayordomo literalmente como un perro. Sus personajes carecen de un 
sano parangón con la figura paterna ${ }^{3}$ y desarrollan un complejo de Edipo tan fuerte y devastador que para poder desactivarlo conviene transformarlo en farsa.

En Accadde al commissariato (Giorgio Simonelli, 1954) Sordi arrestado por ultraje al pudor, desfila con una falda de una manera que ningún otro actor cinematográfico en travesti había hecho antes: es decir, como un hecho natural y obvio, sin ninguna malicia y por eso más perturbador y subversivo: "¿Cómo puede ser indecoroso, señor comisario, llevar una falda por debajo de la rodilla? ¿No la lleva también su mujer?”

Con un simple gag desmonta el mito cansino del latin lover, subrayando cuánta misógina hipocresía se oculta detrás de este. Por ejemplo, en Souvenir d'Italie [Vacaciones en Italia (Antonio Pietrangeli, 1956)] Sordi interpreta a un acompañante dócil y sin dinero mantenido por una mujer madura, muy rica y mandona: es un rol que perfecciona en Racconti d'estate [Sirenas en sociedad (Gianni Franciolini, 1958)], donde es el novio/secretario de una cantante de ópera con sobrepeso con la cual acaba casándose ya que el matrimonio representa el producto último de todas las sublimaciones de cálculos económicos y cinismo. En // medico e lo stregone [El médico y el curandero (Mario Monicelli, 1957)] Sordi aparece en una secuencia única y memorable: un falso superviviente de guerra que tras muchos años aparece ante la antigua novia solo para pedirle algún "dinerillo". Por otra parte también la esposa guapa se puede convertir en mercancía como es el caso de la bellísima Dorian Gray: in Domenica è sempre domenica [Domingo es siempre domingo (Camillo Mastrocinque, 1958)] Sordi empuja a su mujer en los brazos del famoso cantante Achille Togliani para poder participar a un concurso televisivo; en Crimen [Crimen en Montecarlo (Mario Camerini, 1960)] trueca un adulterio por una coartada. Es mugriento y llorón entre Franca Marzi y Giulietta Masina en Fortunella (Eduardo De Filippo, 1957), es un canalla sin excusas ni posibilidad de perdón en su breve aparición en la cárcel femenina de las Mantellate en Nella città l'inferno [Infierno en la ciudad (Renato Castellani, 1959)]. Una sola mujer es capaz de estar a su altura y de atemorizarlo: Franca Valeri, anti-diva y anti-maggiorata que con la única arma

3 El primer verdadero padre será Eduardo De Filippo en Tutti a casa [Todos a casa (Luigi Comencini, 1960)]. 
de la inteligencia consigue dar en la diana. Sus personajes se enfrentan cruelmente hasta la derrota final de /l vedovo [El viudo (Dino Risi, 1959)].

En fin, resulta evidente que en las relaciones con el otro sexo Sordi no cumple con los estereotipos de la italianidad. En una cinematografía donde reina la mitología del Italians do it better, donde machos maduros y ridículos conquistan a chicas jóvenes guapísimas, Alberto Sordi no quiere nunca seducir con el físico o parecer más joven o más guapo. Aceptando el hecho de no ser atlético como Vittorio Gassman o bello como Marcello Mastroianni, el actor romano exaspera su excentricidad física hacia lo grotesco: Guglielmo el dentone [Guillermo dientes grandes] triunfa en el concurso para presentador de telediario y consigue una cita con las gemelas Kessler en I complessi [Los complejos (Luigi Filippo D’Amico, 1965)]; el tenaz paticojo de Brevi amori a Palma di Majorca [Vacaciones en Majorca (Giorgio Bianchi, 1959)] consigue robar a la bellísima Belinda Lee a su musculoso partner norteamericano. Es decir se aleja al máximo de los modelos canónicos de seducción, remotamente distantes del prototipo cautivador de un público femenino que ante la máscara sordiana tendría que sentirse por lo menos incómodo en aquellas ocasiones donde la mujer está en el centro de sus atenciones.

Por lo que concierne al instinto paternal, Sordi va mucho más allá de los tópicos alcanzando la contra-naturaleza ${ }^{4}$ en I/ giudizio universale [Juicio universal (Vittorio De Sica - Cesare Zavattini, 1961)] es un oscuro y despiadado mercante de niños roba a los hijos de los pobres para dárselos a los ricos consiguiendo una considerable ganancia, sin sentir ningún tipo de remordimiento.

Pérfido hasta el final del mundo, desnaturalizado hacia el infinito, difícilmente hubiera podido interpretar el prototipo de los italianos, ni el de algún otro grupo social o comunidad. Sin embargo la revolución estructural y contextual que se verifica a finales de los años setenta en la galaxia catódica aprueba a Sordi como testimonio de la estrategia RAI para la reconquista de un gran número de espectadores perdidos en los laberintos de las cadenas privadas.

4 Cfr. Amelio G., "Sordi contronatura”, en Fofi G., Alberto Sordi. L'Italia in bianco e nero, Milano, Mondadori, 2004, pags. 188- 191. 


\section{El auge de los canales privados}

La fuerza hegemónica había empezado a cojear en 1974 cuando un decreto del Ministerio de Correos y Telecomunicaciones ordena el desmantelamiento de los repetidores que permiten la visión en el territorio italiano de los canales RTSI (Radio Televisión de la Suiza Italiana) y Tele Capodistria, que en los últimos meses habían adquirido gran éxito gracias a su programación en color. Sin embargo la Corte Constitucional, aun confirmando la legitimidad del monopolio estatal sobre las transmisiones vía éter, subraya la ineficacia de una legislación (todavía mussoliniana) incapaz de garantizar la imparcialidad y el libre acceso a la información, por lo que hay que considerar ilegítimo el oscurecimiento de las dos emisoras, pues resulta contrario al principio de libre circulación de las ideas. Poco después Tele Montecarlo (televisión del Principado de Mónaco) emite para una pequeña área de la región fronteriza de la Liguria, sus primeros programas en italiano. Al mismo tiempo, la Corte en relación a la quereIla internacional sobre la clausura del canal por cable Tele Biella $a^{5}$ decreta el principio clamorosamente innovador, según el cual el dominio estatal sobre las telecomunicaciones no puede extenderse a las emisoras por cable que transmiten en un ámbito local, es decir, en un área tan restringida que excluye cualquier peligro de oligopolio. En los meses siguientes se verifica una multiplicación de las emisoras por cable, entre las cuales surge Tele Milano, creada por el constructor Silvio Berlusconi para la futurista urbanización de Milano 2.

Alea jacta est!_El proceso de erosión del monopolio público ha tomado vida. En pleno verano del 1974 Firenze libera comienza sus trans-

5 Primera cadena privada italiana creada en el 1971 por el ex realizador de la RAI Giuseppe Sacchi que consigue el permiso del tribunal de Turín para realizar un telediario de contra-información por cable. Las transmisiones empiezan a finales de 1972, en un momento de vacío de control entre RAl y gobierno italiano (concesionario de las frecuencias vía éter). En un primer momento la cuestión no despierta problemas, pero cuando muchas otras emisoras siguen el ejemplo de los pioneros piamonteses, el Ministerio de Correos y Telecomunicaciones ordena un inmediato desmantelamiento. Pero el propietario Giuseppe Sacchi no se rinde y recurre al Tribunal europeo de Luxemburgo que, después de un primer informe favorable, rechaza el apelo de Tele Biella a favor de mantener una sustancial armonía entre monopolio RAI y comunidad europea. 
misiones vía éter, seguida por un gran número de emisoras privadas que convierten sus cables en antenas. Además, las indicaciones de la Corte Constitucional inspiran la promulgación en 1975 de la ley de reforma de la RAI donde se sanciona la opción estatal sobre todas las transmisiones radiotelevisivas en territorio nacional, a condición de garantizar independencia, objetividad y abertura a las distintas tendencias políticas, sociales y culturales. La nueva ley regula la gestión de la cadena pública y su relación de control con el congreso. Sobre todo se revalida, bajo previa autorización ministerial, la legitimidad de difundir programas emitidos vía éter, en el caso de televisiones extranjeras, y por cable, en el caso de cadenas privadas que no superen un área de 150.000 habitantes. Este último punto será abolido por una nueva sentencia de la Corte Constitucional al año siguiente, de manera que todas las privadas podrán emitir vía éter a condición de que no pongan en marcha interconexiones para transmisiones al mismo tiempo.

Esta última resolución muestra el peligroso olvido de la nueva ley porque no reglamenta el sistema de autorizaciones para el ejercicio de las actividades televisivas: falta de protección del servicio público y de una serie de normas que impidan la concentración de emisoras. De hecho a lo largo de 1976 nacen numerosas cadenas (entre las cuales Quinta Rete del editor Rusconi, Antenna Nord y TV Malta del editor Rizzoli) que en ausencia de un orden jurídico, concurren a la ocupación de las frecuencias. Se inaugura un periodo de desregulación salvaje donde a distintos órdenes de pretores que suspendían las trasmisiones locales inobservantes respondían sentencias de la Corte Suprema en defensa de la libertad de expresión. En este sentido, es emblemática la orden del pretor de Lucca que prohíbe a la RAI invadir las frecuencias precedentemente ocupadas por cadenas privadas, con la emisión del nuevo tercer canal (la actual RAI tre). En diciembre de 1980 el aguerrido conflicto jurídico entre la RAI y Berlusconi (que había ya constituido el consorcio de Canale 5) es mediado por el mismo Ministerio de Correos y Telecomunicaciones, que autoriza la utilización del satélite a la cadena del emprendedor milanés para retransmitir en directo para Lombardía y en diferido para el resto de Italia los partidos del Mundialito: un evento deportivo de fuerte llamamiento, por el cual Berlusconi se había acaparado tempestivamente los derechos de 
emisión. La decisión, aparentemente salomónica, confiere estatuto jurídico al consorcio de Canale 5, que en menos de dos años se transformará en Mediaset adquiriendo Italia 1 de Rusconi y Rete 4 de Mondadori. Pocos años antes de adquirir A. C. Milan y de su sucesiva entrada en la política, aprovechándose de toda una serie de sombras legales y favores masónicos, il cavaliere pone las base de un imperio mediático que es un urticante conflicto de intereses.

\section{Comedia a la italiana traicionada}

En este nuevo panorama la televisión pública se ve obligada a una total refundación en las tácticas de programación, iniciando aquel nuevo paradigma de producción que Umberto Eco llama Neotelevisión ${ }^{6}$. Si en la Paleotelevisión del monopolio público existía una relación paternal entre emisora y telespectadores, ahora la "nueva televisión" apunta a la implicación directa privilegiando la proximidad, es decir, la acentuación de la dimensión de lo cotidiano como retórica del hecho de estar juntos y una estricta relación de confianza, es decir una relación de estricta consanguinidad fundada sobre la auto-referencialidad. El proyecto de Governi se inscribe de lleno en las líneas directivas de este nuevo curso desplegándose alrededor del fil rouge de la empatía. Los individuos comunes con sus vivencias particulares y sus pequeños grandes sufrimientos del día a día son los verdaderos protagonistas de la Historia y tienen derecho a reescribirla aportando cada uno su pequeño fragmento.

A esta misma estructura fragmentada responde la nueva organización narrativa triturada por las interrupciones publicitarias y por el uso indiscriminado del mando a distancia. Instrumento aclamado por la postmodernidad como verdugo de los tiempos muertos y artífice del estilo expositivo de la neotelevisión: el desorden afanoso de las cadenas privadas proporciona material heterogéneo que el espectador-bricoleur reorganiza según el propio criterio de armonía. El principio de fraccionamiento de Storia di un italiano disuelve la oficialidad de los palimpsestos y crea un collage que

Eco U., "Tv, trasparenta perduta", en Sette anni di desiderio, Milano, Bompiani, 2000. 
destruye cualquier distinción entre los lenguajes de la televisión, el cinematógrafo y el documental. Recurrir a los cine-noticiaros del Istituto Luce, antepasados directos de la información televisiva, significa citar el propio universo mediático, la propia trastienda comunicativa. Se trata de un mecanismo de auto-referencialidad, es decir, un modo de reforzar globalmente un sistema lingüístico que intenta conseguir el éxito de una platea que ya tiene una experiencia directa de los hechos explicados. Sin embargo, la mezcla de elementos documentales con secuencias de ficción remite directamente a Paisà [Camarada (Roberto Rossellini, 1946)], o sea, a una de las obras fundacionales del lenguaje audiovisual de la modernidad, filtrada por el espejo grotescamente deformante de la commedia all'italiana que celebra como leader máximo al mismísimo Alberto Sordi? .

El título mismo del programa llama directamente, encausa este subgénero particularmente transalpino, que ha mantenido en auge el dispositivo cinematográfico nacional más allá de los sesenta. Películas como La grande guerra, Tutti a casa, Una vita difficile, (solo por citar aquellas protagonizadas por Alberto Sordi) en las intenciones de Luigi Zampa, Mario Monicelli, Luigi Comencini y Dino Risi querían construir una memoria social de la Historia italiana: una Historia redactada desde abajo, una compilación de estilos de vida, de modas, de creencias, tendencias, mentalidades más que eventos políticos, económicos y militares. Se trata de crónicas paralelas a las oficiales redactadas por protagonistas olvidados, testimonios crepusculares de vicisitudes que quieren contribuir a la formación de una conciencia social: no solo una representación de los eventos, sino una invitación a profundizar, a enfrentarse con las contradicciones de la vida cotidiana.

Recurrir a la commedia all'italiana, reorganizar a través de la lógica del fragmento la representación de un pasado común, responde a una rigurosa estrategia de creación de un target muy amplio: es decir la búsqueda de consenso unánime a partir de un ejercicio colectivo de la memo-

7 Un primer experimento de reconstrucción histórica en llave de farsea había tenido lugar en la película de Luigi Zampa L'arte di arrangiarsi (The Art of Getting Along, 1955), donde el mismo Alberto Sordi interpreta el rol de un auténtico transformista político que consigue estar siempre en la cumbre, cambiando constantemente de bandera y fe ideológica sin ningún tipo de escrúpulo moral. 
ria. La forma misma de enunciación presenta un dispositivo de interpelación que enfatiza metalingüísticamente la implicación humanística. La pulsión de memoria común, el contacto inmediato con el público están cebados por el estereotipo de la italianidad. Se trata de una categoría utilizada como oposición dialéctica a las imprecisas amenazas de la modernidad, del progreso y de las rápidas mutaciones. La misma locución "all'italiana" servía para designar en negativo este constructo como batiburrillo de trazos grotescamente conservadores que las comedias cinematográficas de los sesenta (y parte de los setenta) querían mostrar a través de su mirada deformante y satírica.

\section{Conclusiones}

La apropiación televisiva tanto de los temas como de los modos enunciativos de la comedia a la italiana neutralizan cualquier posibilidad de distanciamiento crítico. Las grandes películas de la época áurea del cine transalpino, convertidas en simple spot de uno u otro editor, pierden cualquier valor cultural. En el enfrentamiento entre cadenas públicas y privadas las obras maestras de la cinematografía nacional son un sencillo instrumento de venganza. Para rasgar la hegemonía de la RAI, construida para una audiencia universal, Berlusconi ha decidido vampirizar el patrimonio fílmico italiano. A partir de los ochenta la programación salvaje, a todas horas diurnas y nocturnas, el nacional populismo casposo insertando el simulacro de Sordi en un spot de una tal marca de espaguetis, así como el simulacro de Totò en una de café, consigue desarrollar una sutil y despiadada forma de control sobre la misma significación: si todo es llevado a la luz, si cada zona de sombra es explorada, se pierde el placer de la reflexión y del silencio. En la reconversión RAI de la antología cómica "a la italiana", se hace referencia a un simulacro de italianidad: una doxa que ha perdido cualquier valor argumentativo; una figura retórica que se traduce únicamente en una imprecisa alusión y una reticencia vacía. 


\section{BIBLIOGRAFÍA}

Amelio, Gianni. "Sordi contronatura". Fofi, Goffredo. Alberto Sordi. L'Italia in bianco e nero. Milano: Mondadori, 2004. 188-191. Stampa.

Bognetti, Giovanni. Costituzione, televisione e legge antitrust. Milano: Giuffrè, 1996. Stampa.

Cesareo, Giovanni. "Le commedie del boom". Spinazzola, Vittorio. Film 1964. Milano: Feltrinelli, 1964. 55-72 Stampa.

Costa, Antonio, Giovanna Grignaffini, e Quaresima Leonardo. Lo spettacolo degli italiani. Strategie di immagine e identità nazionale nella scelta televisiva. Roma: Eri, 1986. Stampato.

Eco, Umberto. "TV, trasparenza perduta". Eco Umberto. Sette anni di Desiderio. Milano: Bompiani, 2000. 163-179. Stampa.

Fofi, Goffredo. "La comédie du miracle". Positif, Vol. 60 (1964). 14-27. Stampa.

Grasso, Aldo. Storia della televisione italiana. I 50 anni della televisione. Milano: Garzanti, 2004. Stampa.

Menduni, Enrico. Televisione e società italiana 1975 - 2000. Milano: Bompiani, 2002. Stampa.

\section{Ludovico Longhi}

\section{ALBERTO SORDI, RAI AND THE NATIONAL IDENTITY OF THE EIGHTIES}

\section{Summary}

By the end of the seventies (the years of lead, full of frustrated and disappointed hopes of social changes) RAI had started a strategic plan to save the national identity. This project was both an answer to an urgent need to reaffirm the hallmark of distinction of the national broadcasting and an attempt to develop a corporate image to be introduced into the new liberalized market of private channels, where the presence of foreign productions had been heavily increased. Meanwhile RAI wanted to play the leading role in the creation of a collective imaginary, broadcasting a series of events like Made in Italy Style, which was a big success thanks to a celebrated Italian designer, or the successful international policy that was the peace mission in Lebanon, as well as the victory in the football world championship in 1982. These positive circumstances awakened interest in defining the concept of Italianness, understood as a socio-political, geo-anthropological and meta-historic category. However, this project brought about a new sense of national 
pride, which succeeded in recycling positively some of the most abused stereotypes of the worst nationalist ideology. Obviously, since only a few of the characteristics of Italianness can be considered exemplary models, it was necessary to start a revision of the Italian comedy satirical types, thus censuring their criticizing power. In this article I want to present an emblematic example of this preventive censure represented by Storia di un italiano, a programme where Alberto Sordi, in order to become the principal icon of Italianness, wanted to tell modern Italian history with some passages of his films, mixed with fragments of the Istituto Luce cine-diary. Nevertheless, the collage, made according to RAI philosophy, ended up diluting the distinctive sarcastic power of Sordi's characters.

Keywords: Alberto Sordi, RAl, comedy, Italian cinema, the eighties. 